\title{
Jaikoa regua
}

\section{Inês de Araujo ${ }^{1}$, Tania Queiroz², Omombe'u va'e mbyapy: Laércio Karai Mirim³}

Kova'e régua: Kova'e nhembopara ma ijayu ha'e ombopara Xadalu Tupã Jekupe pe omboyau ague kova'e mba' eapo ojapo va'e kue kuery, omombe'u mba' eixa pa guembiapo arte regua re ha'e oiko oikoa tetã re ha'e tekoa rupi ha' e maba' eixa vy pa omovaen rã o arte amboae kuery pe, ha'e javive pe oupity aguã rami ojepy'a py rei he'yn re, ha'e javive pe omovaen aguã rami.

Palavras-chave: Grafite. Retrato. Retórica. Trauma.

\section{Transits}

\begin{abstract}
The article comments and transcribes excerpts from the interview conducted by the authors with the indigenous artist Xadalu Tupã Jekupé, relating their artistic interventions and their transit between the cities and indigenous villages to ways of establishing contact between art and its audience through contagion, a gesture that reaches the community inadvertently, and contaminates these societies.
\end{abstract}

Keywords: Indigenous art, activism, urban intervention, graffiti.

1 Universidade do Estado do Rio de Janeiro, São Francisco Xavier, 524 - Maracanã, Rio de Janeiro, R.J. 20943000. E-mail: ines. arj@gmail.com. ORCID: https://orcid.org/0000-0002-7056-1386 Lattes iD: http://lattes.cnpq.br/2689541605877857 . Rio de Janeiro, Brasil.

2 Universidade do Estado do Rio de Janeiro, São Francisco Xavier, 524 - Maracanã, Rio de Janeiro, R.J. 20943000. E-mail: taniaqueiroz3@gmail.com.ORCID: https://orcid.org/0000-0002-0476-9357. Lattes iD: http://lattes.cnpq.br/0370928923427871. Rio de Janeiro, Brasil.

3 Porto Alegre, Brasil. 
Xadalu, arte re omba' eapo va'e tetã py ha'e mbya rei avi, Porto Alegre py hoo va'e, ojapo mba'eapo tetã py nhombyte rupi oexauka va'e mba' ekuaa joegua he' yn rei. Oiko opa rupi ha' e teko ara regua hetaa rupi, imba' eapo ma joegua he'yn he'yn omboaty, tetã régua ha'e tekoa régua, oguenoe mba' ekuaa tetã rupi oiko ague gui ha'e kuery revê guae reve oiko ague gui avi.

Heta regua oexauka, signos ha'e ta' angaa re omoin are oparu pi ombojere jere, heta ombojera ha'e o registro ae tetã mbyte rupi pavê ikuaia rupi, Xadalu tetã rupi omoia rupi mba' emo poética rami oxen, ombojera contrainformação, ha'e mba'epa indígena kuery oikoteven.Oo heta henda rupi publicidade rovai katy, heta mba'e artista oiporu ojapo aguã contato arte re ha'e público kuery revê omovaen aguã, ha'e heta ve pe oupity aguã rami.

Va'e ri, peixa desvios tácitos rami he' yn ju teinko campanha publicitaria py ojapoa rami, estratégia pavê pe omboaxa aguã ma démarche o agi, Paulo Herkenhoff curador ha' e gui critico ema' endu'a rami, "em contaminações culturais do homem nativo concreto, numa interculturalidade atravessada por trocas e resistência a violências". ${ }^{1}$ Ta'angaa, ha'e signos ha' e omombe'u xe va'e artista omonhporandu oaxa rive va'e ha'e hoo va'e kuery, oporandu ha'e ojerure heta henda omoin aguã amboae kuery arandu ha'e kaxo amboae kuery mba'e. Heta pe ombojekuaa ma mba' eapo Xadalu rembiapo iporã hete ve va'e oiko, artista rami anho he'yn oiko, va'e ri oguereko va' e rami, omboaxa ayu heta va'e kuery rami ha'e ogueraa va'e rami nhemikoteven ha'e ayu pavê mbyte rupi, teko ha'e arandu. Omombe'u kaxo ha'e oiko ague ndoexaai va'ekue, imba'eapo ma sensível contingências heta teko re, sociais re ha' e politica oikoa re guare re. ljayu rupi rua py ha'e heta régua rupi ha'e heta oma'e va'e kuery hoo tetã pya rupi, ha'e openaa ha'e oo ojapo aty heta mba'e o soma grafismo revê, adesivos revê, lambes revê, ta' angaa revê, cartazes revê, vídeos revê, pinturas ha'e heta pichações processos de mediação revê. Heta renonde rupi oiko, pichador rami, artista sistema da arte oipotaa rupi avi, amongue py mba'eapo ogueraa va'e, ha'e mba'emo tetã py ha'e tekoa py ogueraa va'e, Xadalu ma ojepy'a py oexauka aguã ta'angaa re oin anho va'e he'yn. Ikyre'yn oendu aguã ha'e kaxo heta regua re, ha'e heta mba'e rexa'a ko yvy re ikuai va'e re, kova'e artista ma pavê mba'e rexa'a ha'e jepy'a py ogueraa.

1 Ver HERKENHOFF, Paulo. "Diagramas de alteridades e trocas", in Catálogo da exposição RSXXI, o Rio Grande do Sul Experimental, Santander Cultural, 2018. 
Amboae kuery pe ayu omboaxa vy ma ou va'e rã re avi onhembo'e mbo'e, imbarete aguã ojapo xe vai va'e kuery rovai, literais ha'e simbólica, agyn revê Brasil py oiko va'e, yvy po kuery ovae hypy'i guive, povos indiegnas kuery rovai ha'e iporiaukue ve'i rovai.

Kova'e arte ha'e ativismo ma oenoin onheporandu aguã identidade indígena agy'in ko tetã mbyte rupi. Ojevale ko heta va'e kuery ijayu va'e gui tecnologia da informação ha' e comunicação kuery omboaxa va'e gui, ha'e openaa ko indígena kuery rami onhemombe' ua re ha'e omombe'u teko regua re ma heta interações sociais ombojera kova'e regua re ikuai va'e.

Terena, ${ }^{2}$ omombe'ua rami, koo mba'eapo agyn arte indígena regua ma oeka heta henda rupi mba' eixa pa ijayu aguã jurua kuery reve oexauka aguã joo rami he' yn nhandererekoa ha'e nhandera' anga oexaukaa ombyte rupi ko o politica ojapo va'e kue nhande mbogue pa aguã história gui. Ko ijayua gue ma omboi entrevista ojapo va'e kue artista revê ara 25 de outubro de $2020 .{ }^{3}$ Koo ijayu gue py roeka ha' e'i gui, Xadalu reve, elaborações artística, espirituas ha' e politicas imba' eapo omombe' u va'e.Mbya kuery ramymino jevy, Xadalu ma omboerya Tupã Jekupe petein imba'ekuaa va'e. Heryma, ha' e pe, ogana voi, oexa amboae rami ma ko yvy rupa.

Tera jypy gua ma omombe'u mamo gui pa reju. Ha'e tera py ma omombe'u mba'e pa rejapo ha'e mba'e pa ndee. Tupã ma, hyapu, oky, yy. Tupã re ijayu ramo jaikuaa ma, joguero'a va'e rã, va'e ri oirun re ijayu rei va'e rã he'yn. Nonhenhomi rei mo'ain, ijayu ete va'e rã. Xerera apy ma Jekupé. Jekupé ma ha'e kuery oenoin, omombe'u va'e rã, onhangareko va'e, hexa pyxo va'e. Etonxe, petein Jekupé hyvy'i ry oin petein imba'e kuaa va'e ramo, imba'e kuaa va'e teinke ojepy'a py omombe'u va'e rã re jekupé pe. Xe ema nin peteingue namboparai tekoa py. Nin peteingue naikotevein ambopara aguã,

2 No artigo "Arte ativista", Naine Terena distingue quatro momentos na história da arte indígena. A autora relaciona esses momentos, que começam com a colonização, às representações construídas no imaginário nacional em relação à presença indígena no país. Se num primeiro e segundo momento as representações construídas por não indígenas evidenciam o controle da imagem dos indígenas, num terceiro e quarto a apropriação das tecnologias ocidentais pelos indígenas torna-se ferramenta de mediação cultural tanto entre indígenas e não indígenas quanto entre os mais novos e os anciões. A autora associa o terceiro momento à criação, em 1987, do projeto "Vídeo nas aldeias", e o quarto, cujo início é datado pela última década do século 20, à intensa interlocução promovida pelas tecnologias de comunicação e informação. Ver TERENA, Naine. "Arte ativista.", in revista ZUM, n. 19, dezembro de 2020. São Paulo: Instituto Moreira Salles. Para mais informações sobre o projeto "Vídeo nas aldeias" é possível acessar o site: http://www.videonasaldeias.org. $\mathrm{br} / 2009 /$

3 Ver Revista Concinnitas, $n^{\circ} .40$. 
xee xo aendu, ja ha'e va'e aema. Ha'e omombe'u ta kaxo hare va'e ramo, xee amoinporã ta noin vei ma py ojevy aguã, xee aikuaa ma. Etonxe petein Jekupe ma inhakuã omoinporã aguã, ngoupive ogueraa aguã, amboae henda py oo vy omombe'u amboae kuery pe. Kova'e ayu nhekambia gui, oxen ta kova'e py ovaen aguã ju. Etonxe xee aiporu kova'e aru aguã arte py ju.

Mitã revê, Xadalu ovaen Porto Alegre py, comunidade Vila Funil py, Alegrete gui ova rire, oxy reve ha'e ojaryi reve, oin porã ve aguã. Hare oiko latinha omono' oa rupi guetarã reve. Kunumia re oiko periferia re vy oikuaa pa ha'e va'e rua ha'e código regua, ojapo aguã pichação tetã py ha'e sticker art regua. Omoin heta sticker indiozinho va'e Porto Alegre py Xadalu, omoin indígenas kuery imba'evende aty yvy'i ry jepi, kova'e ma ogueraa artista onhemboja ve aguã tekoa kuery reve, tape rembe re ikuai va' e reve avi, oma' etyn aguã nda' evei ha'e oin porã aguã va'e reve. Xadalu otende, ha' evy, imba' eapoa arte reve anho'i he' ya, va' eri amboea kuery ojekuaa va'erã avi, ha'e voi oikuaa p ma py periferia py oiko va'e ty vy ha'e perata ndoguerekoi vy, onhepyrun ma tetã re ha'e tekoa rupi oiko oiko aguã.

Anhepyrun aexauka aguã indiozinho ra'angaa aexauka aguã ha'e kuery ikuai ague ha'e py. Petein mba'emo xorive guan, ndaikuaai mba'e etnia pa, ndaikuaai mba'e ve, mba'eve, mba'eve. Tekoa kuery oexa ma vy ha'e va'e vy xereraa tekoa py amombe'u aguã mba'epa oikoa re. Ha'e py avaen ramo iporã raxa, São Miguel py ramo, oin petein tataypy, petein nguaingui'i va'e xevy pe onhemimoin, xeraron, reikuaa pa, xerayu oexauka aguã, ha'e vy ma oreayu katu vaipa. Ha'e vy ma aikuaa xe ve ma mba'e etnia pa ikuai apy Rio Grande do Sul py. Ha'e tein joegua meme py, índio ndee ramo. Yvy po kuery py nomboja'o kuaai mba'emo, heta oin va'e, étnica regua... Amapea vy ma, aikuaa petein pó mboapy etnia ikuai ague va'e ri ha'e kuery nda'ikuai vei ma. Ha'e vy ma aikuaa ve heravy ha'e raingua rire xerembiapo amboe rami.

Kova'e oikoa ma o demarca processo ko mba'emo guio oo ve va'e ju ha'e instalações Xadalu ojapo va'e. Hembiapo ma amboae kuery rami he'yn indígena he'yn oexauka mba'eixa pa indígena kuery ikuaia, ojepy'a py ome' en ju aguã mba'eixa para yvy pó kuery oexa ju aguã ko questão indígena ha'e kuery indígena guia e ju, ha'e kuery ae ikuai ko processo de emancipação re ha'e ojexauka aguã.

Xee vy pe ma mba'emo nhame'e ramo nda'evei rei merami tekoa pe nhamokangy ve merami. Xee 'ame'e ta', kova'e ayu ma hatã rema, ha'e tein mba'eve py nome'e rivei, jaikuaa aema. Ha'e javi oin preço, mba'emo nhe- 
me'e oin preço, nhambo'e heravy py ikangy ha'e ojerure aguã, ojerurea re ikuai aguã. Exami te, oike petein mba'eapo agy'in, xee amboja'o. Petein ojapo ha'e va'e, amboae ojapo ha'e raingua.

Kova'e py, oxen heta questões, mba'e joegua he'yn he'yn realidade pa oaxa, ha'e mba'eixa vy pa ha'e raingua ombovai rã ha'e va'e- tetã py hoo va'e, tekoa py hoo va'e, petein tein remikoteven ha'e artista ha'e kuery pe oupity xea. Xadalu ijayua rema jaexa, joegua he' yn he' yn agencia omoin a Alfred Gell. ${ }^{4}$ Ko mba' eapo ha'e artista, omombe'u vy nhembo'e espiritual, mba'emo kuaa ha'e oiko ague tetã rupi, hoo va'e kuery reve, indígenas kuery revê ha' e huvixa kuery reve, yma guare rami ojevy nha'ã, heta rami ojapo, ojapo petein rede heta ve ouvy va'e ha'e consciência oguereko era vy.

Xevy pe ramo petein mba'eapo opyta porã amboae kuery reve ijayu ramo, ko artista ovaen ve ramo kova'e ayu re, xevy ramo peixa ramo opyta porã, amboae kuery instituição rami he'yn ha'e py oin va'e. Instituição py oin mba aema, etonxe ha'e py oo va'e onhe consolida ve ju... Rua py ma diferente, kova'e omo hatã ve va'e ma pessoas kuery ae, ha'e tein arte tetã regua py pessoas re onhemboja ha'e kova'e ma opyta porã ha'e opyta vai va'e rã, xee arovia ha'e rami. Xee ma a valoriza avi nda'i gutai va'e kuery avi, ha'e tein py nda'i gutai vy py omondoro, reikuaa pa. Va'e ri koo va'e aetu ponto, tetã ý gua ijayu ma ramo xerembiapo re.

Ikyre' yn vy, ko arte Xadalu mba'e ma oeka o intervir reve aguã ha'e heta va'e kuery oexa aguã espaço publico ha'e contato. Intensidade ha'e heta ayu rua py gua.

Rua ma massa, xe aipota massa, xee aipota ha'e javive, ogajara oma'em aguã, xemongavaju, amboae kuery oma'em, jopya kuery ivai, e imagina, ha'e javive huvixa kuery tetã py gua xerexa kuaa, huvixa guaxu ve avi. Ha'e javive xerexa kuaa, 'peva'e aetu tetã omongy'a va'e.

Arte rua py gua ma jajekore aguã rami he'yn, opororo va'e, ta'angaa tuvixa va'e rua py, ipara pa va'e, he'yn vy ta'angaa omondyi paa va'erã, mba'emo

4 Ver GELL, Alfred. "A teoria do nexo da arte", in Arte e Agência. São Paulo: Ubu Editora, 2013. Segundo o antropólogo, a qualidade de agência pode ser atribuída a pessoas ou coisas iniciadoras de sequências causais de um determinado tipo. Para qualquer "agente" existe o "paciente", o objeto afetado de modo causal pelo agente. Seu conceito de agência é relacional. 
direto ha'e gui reto, teinke neakuã, pessoa onheporandu aguã 'Mba'e re tu pe indiazinha i colete prova de bala ju? Mba'e tu oiko? Ha'e vy ma omboayu ta colete prova de bala Guarani Mbyá, ${ }^{5}$, reikuaa pa? Mba'e re tu pe avaxi guaxu oin rua mbyte py? Mba'e tu oiko?

Ome'e vy ayu hare okyririn va'e kue pe, artista nhane moma' endu'a Porto Alegre ojapo ague tekoa ary rive ha'e imba' eapo ojerure yvy ha'e kuery mba'e ae. Kova'e nhorairo regua, heta intervenções mba' eapo Area indígena va'e omonheporandu ko omanda va'e ha'e ko território re. Ko Area indígena, heta va'e avi, ojekuaa, hare re avi, heta regua, ojekuaa ma ojegua reve, lambes py, heta suporte rupi ha'e heta henda rupi, peixa, kova'e rami, bandeira rami, omoin jave aste cúpula Museu da Arte do Rio. ${ }^{6}$ py.

Rua py ma oiko ypy'i Area indígena, ojexakuaa va'e ha'e okanhy va'e kuery reve. Oiko ypy gui ve, ko mba'eapo ojekuaa cartaz hovy rei va'e ha'e iju va'e rami $40 \mathrm{~cm}$ x $40 \mathrm{~cm}$ heta ve ha'e opa rua rupi. Kova'e ayu cartaz re "Atenção Area Indígena", nhane moma'endu'a placa de transito re, nhane mo porandu questões de território re. Ko sinal omoen, va'e teinko, nane moma'endu'ai yma mba'eixa pa ko indígena kuery oiko ague yvy re. Ha'e he'yn re o sinaliza transito ae ha'e petein henda re nhomo tare'ya, oexauka va'e nhomo tare'ya espaço ha'e gui simbólico tetã py. Area indígena ma omombe'u avi peixa mba'eixa pa indígena kuery pe oguereko axya comerciante kuery, loja já kuery, tetã py ikuaia ja re omba'e rei rei ovende aja.

[...] xee anhepyrun amba' eapo aguã petein henda kyrin va'e py, tetã py ma jareko petein henda kyri'in va'e ha'e nhorairon petein henda ha'e javi cultura reve, petein amboae pe omokomba xe ve, ha'e ikangy ve va'e ma oendu ve ta, ha'e onhemoin ta tenonde ve. Jaexa ramo petein mbya centro de Porto Alegre py omba'e rei rei ovende xe ramo ha'e tenonde ve onhemoin xe. Oiko xe ve vy ha'e py oin. Va'e ri jaikuaa aema jurua kuery reko Heta vea, fisicamente ha'e gui estruturalmente, ha'e gui culturalmente, ha'e va'e re apensa raxa.

Ojopy jeve processos jajexaukaa ferramentas tecnológicas rupi ma ojekuaa riae Xadalu mba' eapo re. Omba' eapo ojapo indígena kuery oexaa rami, onheporandu aguã mava'e pa o representa ha'e mava'e pe avi,

5 Comunidade indígena Guarani Mbyá.

6 Xadalu no MAR, março de 2020. 
omoin va'e kue petein mba'e tuvixa rai va'e oo guaxu Caramelo FAU/ USP py, mamove gua rami he'yn, omoin heta adoção estratégicas estéticas regua, ayu gráfica regua re ha'e fotomontagem, ha'e javi ambiente tetã regua ha'e espirituais. Peixa mbya kuery iparaa regua ha'e cores vibrantes, ta'angaa huun va'e ha'e xiin va'e, mboapy huvixa ve indígena kuery mba'e sul pygua, mokoin avakue ha'e petein kunha va'e, ojekuaa ve, va'eri omoin porã mba' emo yma guare, montagem de fundo ma, ipara joo rami meme ha'e ojeguaa vibrante,o transborda ha' e overa oiko ve va'e rami ha' e javi henda rupi.

Kova'e ta'angaa rema iparaa oexauka cosmo petei tein mba'e ha'e gui mba'eixa pa hete oin, xee anhembo'e rema va'e kue, ijayu ha'e va'e pessoa re. Etonxe Dionísio ma ijayu porã va'e, ha'e mboruvixa va'e kue, ha'e yvate katy okuan ve'em; amboae, ma Mariano, oma'e tyn va'e, ha'e ma oexauka va'e indígena kuery ikuai rive va'e he'yn a, ha'e omba'eapo yvy re, yvy já, vy ma ha'e axara reve oin, ha'e gui, Laurinda ma, nguainguive va'e ha'e javive oexa kuaa va'e. Xee ajapo peteim aó ritual py gua ha'e pe, ha'e oguenoe ngua'angaa takua pu opoxi'a py reve, takua pu mitológico reve, kova'e [oexauka takua pu]. Kova'e takua pu ma nhanderete ikaren va'e ombo'y vi. Ha'e oguenoe ngua' angaa kuery, ha'e rire ma oin petein mba'eapo grafismo re rire ha'e va'e mba'emo ojekuaa espaço py, rire xee ajapo mboapy ta'angaa ha'e va'e regua, recurso FAUSP mba'e re, ha'e va'e ta'angaa oguereko, aikuaa ramo $12 \mathrm{~m} \times 5 \mathrm{~m}$.

Peixa mba' emo pyau jurua kuery mba'e oiporu tein ma indígena kuery remikoteven re, ha'e va'e jeapo joo rami he'yn he' yn. Kuaxia para Xadalu omboayu va'e kue Invasaõ colonial Yvy Opata, yvy opa ta, paven ruvixa apy Rio Grande do Sul py gua, Mboruvixa Tenonde Cirilo Karai, onhemoporandu:

Kova'e agyn rami he'yn, mboka tata revê gua va'e kue, agyn jojuka mba'e mo pyau py haxy oendu he'yn re, ovy'a okuapy merami, ouga joupive vy ovy'a okuapy, va'e ri opa'i apy ojekore pa rive, ore rojojuka ore meme ha'e kova'e mboka ma ivaikue ve va'e.Peixa gua oexauka va'e ma kuaray oike ramo, opy'i py ou ra'angue va'e ri, kunumingue vídeo game ouga aguã rive onhemboaty okuapy,

7 O trecho do texto do cacique Mburuvixá Tenondé Cirilo Kara foi extraído do folder da exposição Invasão Colonial ÏVY OPATA, a terra vai acabar, realizada em Porto Alegre, no Museu de Arte Contemporânea do Rio Grande do Sul. 
Ha' e rami avi oexa, artista ijay omba' eapo py, oin va'e ha'e va'e exposição py ae, va'e ri arapuca rive aeri televisão oin apy, oexauka filme petein sala py 39 indígenas Free Fire py ouga va'e, vídeo game imboka ra'angaa va'e.

"Ha'e kuery joexa ara py petein pó mboapy hora pyavy jave, mboapy pó tekoa reve, ouga aguã mbya py ijayu va' e meme. Petein televisão oexauka, xee amoin porã mba xee anho' in aepy oje'a kue ha'e kuery ijayua, 'esquema py'. Etonxe reuga reve ndeayu, 'eju apy, tereo ha'e py', mbya py meme, ha'e kuery ra'angaa, indígena kuery, mba' emo rague reve, mboka revê. Peixa gua invasão colonial he' yn a ma xee ndaikuaai,mba'emo pyau jurua kuery mba'e tekoa py iporã ve era vy, ha'e kuery joexa riae ara ara re, oin campeonato peixa guare re perata o vale va'e, xo mbya kuery."

Oficina ojapo ha'e omboja' o mba' eapo ovende va'e kue gui, onhekoteven ve artista oexauka aguã onhetende ve aguã, omombe' u va'e, omoin aguã pavê tekoa py gua kuery. Oexauka aguã omba' eapo otende aguã indígena kuery amboa kuery mbya ijayu he'yn va'e kuery no tendei aguã.

2018 re oenoin ramo curador ha'e critico Paulo Herkenhoff, Xadalu oexauka omba' eapo Arqueologia do presente, mbya py ipara va'e petein pichação parede 5 metros oikea yvy'iri Museu dos Direitos Humanos do Rio Grande do Sul gui. Ko ayu re ojepy'a py aguã onhepyrun kova'e mba'eapo oma'e efeitos da marginalização re vy, kyringue indígenas kuery oaxa va'e kue re, oxy kuery omoin run vy tetã re ao py.

Xee aexa kyringue oxy kuery pe omoin ru vy oo va'e, mbya reko ae ha'e va'e ha'e va'e te ae, ha'e kuery oo ja ha'e va'e aema. Kyringue tetã py oo vy opa- nhande ndajaexai, va'e ri ha'e oxen marginal, ha'e oikuaa pa ta py tetã iporã va'e he'yn, ha'e oexa ta, jaexa vy py nhanhembo'e avi, rãke?... Mba'e tu oiko ta koo sistema marginal py oiko va'e re, rua py oiko py sistema marginal py aema oiko. Rejerurea re reiko vy py sistema marginal py ae reiko. Xee anhepyrun ma aexa aguã amboe kuery no tendeia tekoa py gua ijayua ha'e tein tekoa py gua jurua py nda'i jayui py tetã py oiko vy, ijayu mbya py rive.

Xee aexa ha'e kuery ayu na tendeia, no tendei ijayua va'e ri o marginaliza. Petein ara, ha'e kuery marquise guyry ikuai, oky ha'e jave ikuai, pichação guyry. Ha'e ramo ha'angaa anoe, ha'e vy amombe'u 'mba'e tu koo va'e ha' e? Pichaçao ha'e. No tendei vy ijayu: 'ndee vy pe ha'e ve va'e ramo, etonxe ha' e ve.' Xeayu: 'kova'e ma petein arte ojapo oexaa aguã o protesta ramo, território o demarca aguã, mba'emo.' Vy ma ha'e va'e ha'e py opyta rive. 
Ha'e vy ajevy ju ateliê py ha'e rire mbovy pytun ndakei ha'e va'e re apensa $v y$. Ha'e rire petein ara xeayu petein pichador gangue py gua yma guare reve - ha'e kuery oenoin gangue rami - , xeirun raxa, ha'e ijayu: 'e imagina nhambo'e va'e rire pichação tekoa py, ha'e gui jajapo va'e rire petein painel pichação mbya py museu py'. Ha'e vy ha'e: 'Any, any, kova'e apensaa rami he'yn, kova'e ma amboae regua, nin ha'e kuery voi no tendei mo'ain peixa gua.

[...] xee voi xe guta xeayu anhetengua re ramo no tendeai ramo, va'e ri anhetengua. Reikuaa, xee amombe'u xe anhetengua ndee nere tendei va'e, amboae ayu py, va'e ri anhetengua. Ndee repensa opa mba'e, va'e ri nere pensa mo'ain ijapu pa terá anya re, Repensa ta opa mba'e re, va'e ri anhetengua ma omombe'u ha'e va'e ha'e mba'eapo anhetengua ja ha'e va'e aema.

Oaxa espaço, yvy gui teto peve, inscrição tuvixa rai va'e, mbya py ipara va'e, instituição renonde py oin. Mba'eapo oin hatã petein inscrição yvy rupa o opera de forma negativa. Amboae henda rupi, xee aexa ramo, petein nguainguive va'e omombe'u va'e kue jurua kuery ovaen ague ijyvy py ha'e hetãra kuery apy; petein henda iporã hete vea ma ijayu ague nda'e vei omombe' u aguã ha'e omboaxa aguã, oin oiko ague re ha'e nda'e vei omboaxa aguã oiko ague.

Oma'e va'e kuery ma ha' e javive, amboae henda rupi ma, ha'e va'e, onhoconfronta avi expressões do incomunicável revê ha'e oiporu vy omboayu kuaa kuery renda. Oexauka vy omboayu kuaa va'e he'ya, omoin porã vy henda patrimônio público py, oexa va'e kuery ha'e vy oexa ijayu porã va'e nonhendui ramo o residi ramo margens heta ve va'e kuery o forja ramo espaço de partilha patrimônio comum mba'e, Ocasião vernissage jave, petein grupo indígena oipou museu. Ha'e kuery anho'i omboayu kuaa vy ipara va'e, ha'e kuery omombe'u mba'e pa ipara va'e ijayu jurua kuery pe. Ogueraa puku he'yn re, Arqueologia do presente ma ombopara questão indígena urgência py. Guerra colonial jave re, ndoexa xei inscrição histórica apy jypy re ikuai va'e kue oiporu py jurua kuery ikuaaia renda, sociedade brasileira ma agyn gua ha'e rami vy oipytyvon

Ombogue aguã ha'e omokyririn aguã "nda' evei nhapensa aguã ijapua", teinke oiko ve jajo'o aguã ha'e nhamboayu aguã. Opa'i apy ma, xadalu ae oexauka mba' eixa pa ha'e otende arte regua ha'e opa rupi oikoa re, otendea tempos kuery ikuai va'e ha'e mba' eixa pa ha'e kuery reve ikuai rã. Iguto rei jaikuaa ramo noin contradição personagens kuery oiko va'e reve, nin amboe kuery relação complexa petein tein mba'e reve. Arte, 
mba' eapo pavê pe, comunicação, contaminação, circulação, sobrevivência. Va'e ri apy ma onhetende joo rami he'yn he'yn oin nhekoteven, ha'e vy ma, atitude joo rami he'yn rã.

Agyn ma xemba'eapo, pesquisa he'yn ae jepe, va'e ri ha'e javive anhetengua ha'e kaxo ha'e mitologia reve, petein mba'e mo iporã ete va'e, imba'e rexa'ã va'e ae ha'e rami oexa. Ha'e kuery oexa ha'e va'e mba'eapo, ha'e vy ijayu anhetengua ha'e iporãa. Va'e ri ha'e tein ndajaipotai ícone rive oxen, kova'e xeayu xepy'a py reo in va'e. Ideia ma tekoa pe amoky re'yn nda'ei xevy pe xerekuaa aguã, ha'e kuery voi onheporandu aguã. Ha'e tein jaikuaa aepy mbya kuery jegua he'yn ae oguerekoa, jaexa oje'a je'a va'e oin a, xee aexa jepy'a py reve, xee aexa arte indígena tecido rami, já amboae rami ma, ja oje'a ma, petein miscigenação, petein mba'e jaexauka aguã ha'e jaru aguã nhandereko iporã va'e, ha'e tein pave onheporandu va'e py, oguereko...

Xe cosmo astral pyma Tupã gui gua, nin oguereko ete va'e paciência tempo reve, ha'e kuery ojexa vai tempo reve, ojexa vai ete tempo reve. Ha'e vy ma tempo rua py gua xe mo ancioso raxa ha'e tein rua py re resolve pa reve py mba'emo, confusão oxen ta ramo oxen reve py, acola ta ramo acola reve py, akanhy ta ramo akynhy reve. Etonxe a resolve reve py ha'e va'e situação. Tempo ateliê py gua ma tempo tekoa py gua rami avi, tempo de incubação rami. Etonxe nda'evei, petein ideia oxen porã aguã, teinke tempo oin. Etonxe, tekoa py, ha'e kuery ijayu jepi ko tempo xemba'e apy tekoa py gua he'yn ae, xee tempo apy gua he'yn, pessoa física rami, kova'e ma xe tempo, tempo inhakuã va'e, ha'e kuery oenoin tempo perecível rami. Kova'e tempo ma amboae rami, tempo ateliê py gua rami, xee tempo ete, etonxe ha'e kuery oguerovia ndee remba 'eapoa amboae tempo py ju ore ma ronhembo'e romba'eapo aguã tempo ore vida py ju. Xee areko noção tempo ateliê py gua xe tempo interno a rami, petein tempo ijaju aguã ideias oin eravy aguã, etonxe jajapo eravy ideias oin aguã ha'e vy oxen eravy. Va'e ri ha'e raingua xee anhembo'e eravy, agyn, parte intelectual py, cosmologia py. Anhenhandu, anhenhandu ete jave xee a rua py acola vy rire ajevy ju. Xee ajapo xe artes visuais aiko ve va'e re, relação tekoa ha'e rua reve. Va'e ri nda'evei ajapo aguã ha'e tein xee py fator social py gua, reikuaa pa? Etonxe tekoa py aa jave xeayu vy, teinke reo riae, xee mbovy'i apinta, ha'e [huvixa] ijayu 'repensa ramo nhi'ã re pinta rive ta, reike apy rire nhi'ã re pinta rive ta.

Xee xeayu: 'Hen, xee apensa apinta rive ta ha'e gui acola rive ta.' Ha'e: 'kova'e ko ndoikoi. Kova'e py oike va'e ma, amboae rami oike. Etonxe nda'evei re pinta rive aguã, porami ijayu... 


\section{Referências bibliográficas}

Entrevista com Xadalu Tupã Jekupé, in Revista Concinnitas, n. 40.

GELL, Alfred. "A teoria do nexo da arte", in Arte e Agência. São Paulo: Ubu Editora, 2013.

HERKENHOFF, Paulo. "Diagramas de alteridades e trocas", in Catálogo da exposição RSXXI, Rio Grande do Sul Experimental, Santander Cultural, 2018.

KARA, Mburuvixá Tenondé Cirilo, in folder da exposição Invasão Colonial ÏVY OPATA, a terra vai acabar, MACRS, Porto Alegre, Rio Grande do Sul.

TERENA, Naine. "Arte ativista.", in revista ZUM, n. 19, dezembro de 2020. São Paulo: Instituto Moreira Salles. 